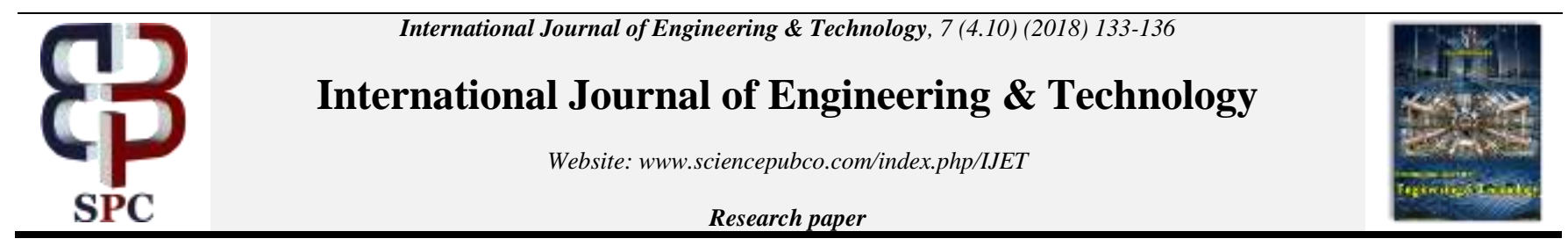

\title{
Analysis of Component based Computing
}

\author{
P. V. Kumaraguru ${ }^{1}$, V. J. Chakravarthy ${ }^{2}$, M. Seenivasan ${ }^{3}$ \\ ${ }^{1,2}$ PG Department of Computer Applications, Guru Nanak College, Chennai, Tamil Nadu, India. \\ ${ }^{3}$ Department of Mathematics, Annamalai University, Annamalainagar, Tamil Nadu, India. \\ *Corresponding author E-mail: emseeni@ rediffmail.com
}

\begin{abstract}
To achieve a precise goal of components on different platforms that are presented the some components in order to co-operate with one another over a communication network. The component should be able to access services provided through remote, location transparent service in vocations. The major role of component-based method is represent an ideal framework for component-driven in client/server computing. One of the good implementation examples of broker architecture is Common Object Request Broker Architecture (CORBA). The component based technologies discuss the proposal of distributed object of CORBA which is the Object Management Group's (OMG).This paper proposes the broker architecture as CORBA has distributed system that can be demonstrated by client-server architecture which practices the base for multi-tier architecture.
\end{abstract}

Keywords: Component based, Common Object Request Broker Architecture (COBRA), Object Request Broker (ORB), Object Management Group (OMG) and Distributed Computing Enviornment (DCE).

\section{Introduction}

The basics of component technologies and client-server computing propose the framework using the distributed objects. This paper focus on the delegation between the client/server framework and stratification on client/server system into levels [1].CORBA is developed by Object Management Group (OMG) which is an industry standard of more than 700 groups of companies to support in programming distributed objects and also not a programming language. The architecture of CORBA is created on the object model. The OMG is used in Object Management architecture guide, where the model is derived from the abstract core of object model which is not directly realized by any particular technology. This allows applications to be built in a standard manner using basic building blocks such as objects. Hence CORBA is separates the client (request of services) from the server (provider of services). The system is based on the collection of objects by welldefinite encapsulating interface. CORBA object is significant to differ from typical programming objects.

CORBA objects run in three different ways [2].

- It can run on any platform

- It has Interface Definition Language (IDL) mapping that can be written in any language.

- It can be located wherever on the network.

In this paper Object Management Architecture (OMA) tries to define the various high-level facilities that are necessary for distributed object-oriented computing. This mechanism of OMA is the ORB that provides the core object location transparency, activation and communication. The CORBA specification based on the OMA which offers description of the facilities and interfaces that provided essentially by yielding ORB was released.

\section{Literature Review}

Various component infrastructures use different security standards CORBA is based on Component Model (CCM) is defined by the Object Management Group's standards which may use the security services for implementation [3]though DCOM and .net is built on a dissimilar standards[4].CORBA security architecture which provides by CORBA security service that can support to meet different needs of variety of security policies. The principles of authentication and specification which is defined by security functionality and infrastructure based on access control and authorization [5]. However the applications of component based security standards is not yet entirely investigated on the effect on the scalability performance. DCOM is just like CORBA which it is efficiently splits the interface from functionality using on IDL. Microsoft has preferred to use IDL which is based on Distributed Computing Environment (DCE). The IDL is nothing but neither CORBA nor DCE complaint; this rigorously confines the potential for interoperability. In addition Microsoft's object linking and embedding technology which separate the interface functionality is provide by using the Object Definition Language (ODL). DCOM doesn't support the object of traditional notion and don't have a state, moderately they are collections of interfaces. By suggesting the DCOM objects, one could liken to collection of algorithms which are inherently not a prevailing computing machines as CORBA objects [6].A distributed system forms the base for multi-tier architectures which can be demonstrated by clientserver architecture, alternatives are CRBA is the broker architecture and Service Oriented Architecture (SOA).This type of architecture processing information is not confined over some independent computers to a single machine rather it is distributed. There are various technology frameworks which is used to support the distributed architecture such as .NET web services, CORBA, .NET, J2EE, AXIS Java web services and Globus Grid Ser- 
vices. This development of distributed application which supports appropriately middle ware infrastructure. It offers a buffer between the network and application [7]. Androutsellis-Theotokis and Spinellis [9] Surveied of peer-to-peer content distribution technologies. Koh and et.al [10] computed an analysis of performance interference ef fects in virtual environments. Ghemawat and et.al [11] studied the google classification system. DeCandia and et.al [12] calculated amazon's extremely accessible key value Store. Hanemann and et.al [13] analysed a service familiarized design for multi-domain network observation in ICSOC. Foster, and et.al [14] performed a met computing infrastructure toolkit. Blum and et.al [15] derived grid resource allocation and management exploitation procedure economies. Papazoglou and et.al [16] studied service familiarized architectures. Petersen and et.al [17] disscused replicated information services for World-Wide Applicationsr. Orfali and et.al [18], [20] and Chappell et.al [19] studied the Essential Distributed Objects Survival Guide.

\section{Components of Broker Architectural Style}

The components of broker architectural style are discussed through following heads

\subsection{Broker}

The entire document should be in Times New Roman. The font sizes to be used are specified in Table 1. In the case of forwarding and dispatching of results and omitting in coordinating communication as the responsible for broker which is an invocation oriented service. The client has sent a message with the aid of message or document oriented broker. The responsible of the broker is by servicing the task such as service request, transmitting request, responding back to client and maintaining the suitable server. These can also retaining the information of server registration which include services, functionality and also location information. In addition, it support API's for requesting client, servers to respond, transferring message, server components in registering and unregistering and location of servers.

\subsection{Stub}

This is produced during Static Compilation time and after it is organized to the client side that is used as proxy for the client and act as an intermediary between client and broker. This provides an additional transparency between broker and client. The inter process Communication (IPC) were hided from proxy during the level of protocol and also performing marshaling in parameter value and un-marshaling in results from server.

\subsection{Skeleton}

The service interface of compilation is produced from skeleton and it organizes to the server side that is used as proxy for the server and it encases the specific network functions for low level system. This is provided for high level APIs which act as intermediary to server and broker and also perform like receiving request, unpacking of request, method of argument is un-marshaled, call the appropriate service and marshaling of result before it send back to the client.

\subsection{Bridge}

The two different network located in various communication protocols are connected by using bridge. It intermediary various broker which include Java, CORBA, .NET remote and DCOM broker. IT is an optional component that hides the detail of implementation during two brokers perform and also considering parameter and request of a format is translated in to another format.

\section{Broker Implementation in CORBA}

It is middleware solution with an international standard for the Object Request Broker (ORB) which provides communication among the object distributed that is defined by object management group (OMG).

\subsection{The Object Request Broker}

The Software that implement middleware COBRA specification is said to be ORB which act as the heart of the COBRA has responsible for essential mechanism to perform the task

- To find the object implementation in order for requesting.

- Preparing of object implementation for receiving the request.

- To make up the request, data is communicated.

Figure 1 shows the CORBA architecture[1]. The major ORB components used by the client and the implementation of the object are shown.

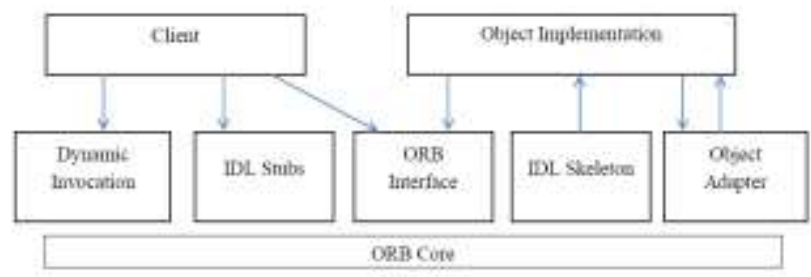

Fig.1: Shows how the five major components of CORBA fit together.

The object of CORBA may exist everywhere on the network in which the locality is entirely transparent. Details of the object like language according to written object or operating system that currently run have hidden to clients while implementing an object there is no other consideration involved in object except interfacing of object during the selection of serving object. The fundamental system and application services provided from CORBA to the object is completely depend on it for management. The involved services in the level of middleware have eliminate the requirement for more "Mix in Classes" were those classes have deformed the object model and hierarchy of the system get complicated groundless[8].The ORB provides a mechanism for transparently communicating client requests to target object implementations. The complication of remoted object communication which is dealed based on the requirement of client get shielded by using ORB, it handle the difficulties while coordinating the task. The CORBA 2.0 specification mandates inter-vendor ORB compatibility, which is accomplished via the required Internet Inter-ORB Protocol (IIOP). IIOP provides a common communication backbone between different ORBs by adding several CORBA-specific messages to the TCP/IP schema already widely used today. Most of the middleware services are provided with ORB were the distributed object system get robust. Several features of CORBA are fatigue from determined model like Message-Oriented Middleware and Remote Procedure Call(RPC). In order to establish a client/server relation with component ORB is used in CORBA. The ORB intercepts method invocations from client objects and routes to an appropriate server. The services needed to convene the demand request of generic client. Based on the details of implementation from the programmer and run time variable get shielded by CORBA as the capability using ORB. The ORB will not bind the provided component to a role of client/server the similar component perform as a client for other object as the heart of CORBA's stable interoperability is the interface definition language (IDL).CORBA IDL stubs and skeletons serve as the "glue" between the client and server applications, respectively, and the ORB. The transformation between CORBA IDL definitions and the target programming language is automated by a CORBA IDL compiler. The use of a compiler reduces the potential for incon- 
sistencies between client stubs and server skeletons and increases opportunities for automated compiler optimizations.

\subsection{The Object Management Architecture (OMA)}

OMA is subsequently higher level which frames CORBA architecture. The OMA goal is to admit application to serve their fundamental function through usual interface. The OMA contains CORBA facilities and CORBA service are the two major components which is shown in figure2.Based on the application level, higher level of functionality is provided is CORBA facilities. The basic services which are essential for object inclusive of event services and name services were provided from CORBA services. Furthermore CORBA facilities are divided into two, they are horizontal and vertical CORBA facilities. The functions like system management, information management, user interface and task management are provided in the horizontal CORBA facilities. Vertical CORBA facilities which are based on domain functionality provided for the particular domain like health care, telecommunication and electronic commerce.

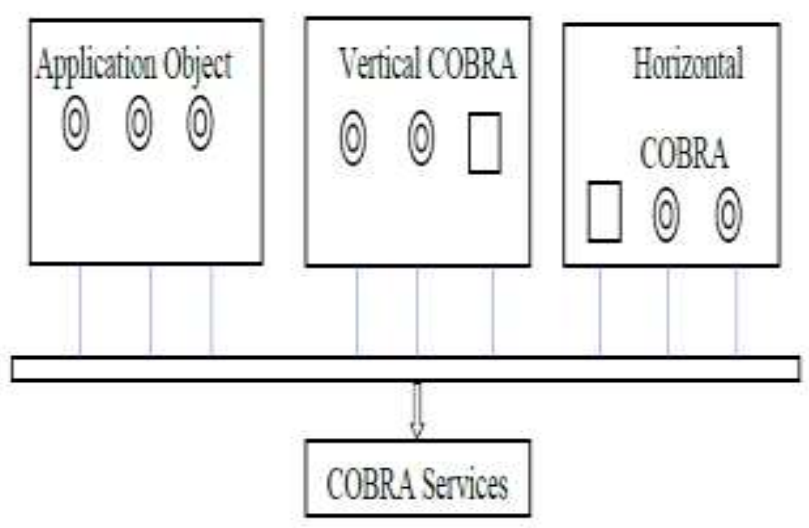

Fig.2: Object Management Architecture (OMA)

The widely available object services are get interfaced which are generally used to support the splendid application is the environment of distributed object create on the CORBA compliant ORB The OMA object services have the following features mentioned below

- Few or several object services are used for object.

- Object service Operations are specified in IDL.

These services are said to be CORBA services. Some of the object services are discussed below.

- Object naming Service

This service is forever specifying the relative to the naming context, which support in association with name-to-object is known as name binding. At the same time various name can be bounded to an object for same and various context. This service support enormous operation like bind, look up and unbind.

\section{- Event Service}

The notification of event based on the interested object is supported in these services. The asynchronous communication is provided between co-operating and remote objects.

\section{- Persistent Object Service}

These services have provided with general interfaces for mechanism used for managing and retaining the determined state of object in a data-store independent manner. Of course, the object has the responsibility of managing its state, but it can use or delegate to this service for the actual work.

\section{- Concurrency Control Service}

In this services, object intermediately simultaneously access more client, so the object is accessed that remain coherent and consistent.

\subsection{New Features in CORBA 3.0}

CORBA had to evolve to remain viable as a basis for distributed applications. As part of this continuing evolution, several significant new features are being added to CORBA that will be part of CORBA 3.0. The new features include Portable object adapter (POA), CORBA messaging, and objects by value.

\section{Conclusion}

CORBA's object references provide a clean way of gaining an object's interface. Callbacks allow servers to control clients and allow clients to receive new content to add to compound documents. CORBA can able to admit multiple source which is to be encased and server pool also be built for supporting 3 tier client/server system. The functions support self-describing, easy interoperation and admit flexibility in binding are made through CORBA component by providing dynamic discovery of object interface. The advancement of omnipresent middleware is accessible on entire platform which will advance to accurate location transparency and CORBA state the open and wide standard that ensures the continuous innovation and evolution of the system. In order to improve the speed of CORBA ORB is interoperating with object c11 and then integrated with java.

\section{Acknowledgement}

First, we thank the almighty for giving us a strength to have knowledge in this topic and made me to frame this paper. Secondly, we thank Capt. Dr. S. Santhosh Baboo, Registrar, and Mononmaniam Sundaranar University, who motivated us towards research fields.

\section{References}

[1]. Scott M. Lewandowski, "Frameworks for Component-Based Client/Server Computing", Vol. 30, No. 1, March 1998.

[2]. D.G.Schmidt and F.Kuhns, "A Overview of the Real-time CORBA Specification", Volume: 33, Issue: 6, June 2000.

[3]. N. Brown and C.Kindel, "Distributed Component Object Model Protocol-DICOM/1.0”,InternetDraft,24-January-1996. http:// www.Microsoft.com/oledev/olecom/draft-brown-dcom-v1-spec02.txt.

[4]. U. Lang and R.Schreiner, Developing secure Distributed systems with CORBA, ArtechHouse, February 2002.

[5]. Wegner, Peter. "Why Interaction is More Powerful Than Algrithms." Communications of the ACM, May 1997, 80-91.

[6]. Dean J, Ghemawat S. Mapreduce, " Simplified Processing on Masive Clusters", in OSDI'04,2004.

[7]. Olston C, Reed B, Srivastava U, Kumar R, Tomkins A, Pig Latin: A Not-so-Foreign Language for Knowledge Process, in SIG MOD '08: Proceeding of the 2008 ACM SIGMOD International Conference on Management of Information, New York, NY, USA: ACM; 2008. P. 1099-1100

[8]. CHAPPELL, D, Understanding ActiveX and OLE. Microsoft Press, Redmond, WA, 1996

[9]. Androutsellis-Theotokis S, Spinellis D. A Survey of peer-to-peer content distribution technologies, ACM Computing Survey 2004:335-3771.

[10]. Koh Y, Knauerhase RC, Brett P, Bowman M, Wen Z, Pu C, An Analysis of Performance Interference Effects in Virtual Environ ments, in ISPASS. IEEE Laptop Society; 2007. p. 200-209.

[11]. Ghemawat S, Gobioff H, Leung ST, The google classification system, SIGOPS oper. Syst Rev 2003:37:29-43.

[12]. DeCandia G, Hastorun D, Jampani M, Kakulapati G. Lakshman A, Pilchin A, Dynamo: Amazon's Extremely Accessible key value Store, in SOSP '07. New York, Ny, ACM: 2007. P.205-220.

[13]. Hanemann A, Boote J W, Boyd E L, Durand J, Kudarimoti J Lapacz R, In: Benatallah B, Casati F, Traverso P, editors. Perfsonar: A Service Familiarized Design for Multi-Domain Network Observation in ICSOC, Ser. Lecture Notes in Computing. 
[14]. Foster I, Kesselman C. Globus: A Met Computing Infrastructure toolkit. Int J Mainframe Appl 1997;11(2):115-128.

[15]. Blum M, Floyd RW, Pratt V, Rivest RL, Tarjan R.E, Grid Resource allocation and management exploitation procedure economies. Grid Computing 2015;102:10.

[16]. Papazoglou MP, Heuvel W.J, Service familiarized architectures: Approaches, Technologies and analysis problems VLDB J 2007: 16:389-415.

[17]. Petersen K, Spreitzer M, Terry D, Theimer M, Bayou, "Replicated information Services for World-Wide Applications", in Military Action 7: Proceedings of the $7^{\text {th }}$ Workshop on ACM SIGOPS EuroPean Workshop. New York, USA: ACM: 1996.P.275-80.

[18]. Orfali, R., Harkey, D., and Edwards, J, The Essential Distributed Objects Survival Guide. John Wiley, New York.

[19]. Chappell, D, Understanding ActiveX and OLE. Microsoft Press, Redmond, WA, 1996

[20]. Orfali, Robert, Dan Harkey, and Jeri Edwards, The Essential Client/Server Survival Guide. New York: John Wiley \& Sons, Inc. 1996. 